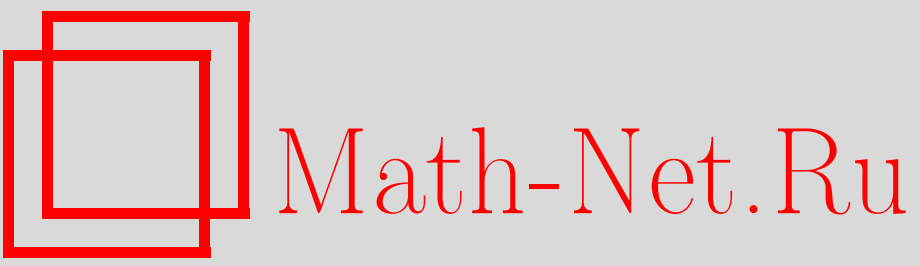

Е. М. Аристова, Некоторые подходы к решению задач линейной многокритериальной оптимизации, Итоги науки и техн. Сер. Соврем. мат. и ее прил. Tемат. обз., 2021, том 190, 3-13

DOI: https://doi.org/10.36535/0233-6723-2021-190-3-13

Использование Общероссийского математического портала Math-Net.Ru подразумевает, что вы прочитали и согласны с пользовательским соглашением

http: //www.mathnet.ru/rus/agreement

Параметры загрузки:

IP: 3.82 .47 .9

26 апреля 2023 г., 12:44:48 


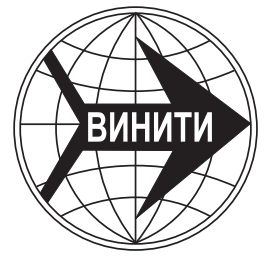

ИТОГИ НАУКИ И ТЕХНИКИ.

Современная математика и ее приложения.

Тематические обзоры.

Том 190 (2021). C. 3-13

DOI: $10.36535 / 0233-6723-2021-190-3-13$

УДК 62C86, 90С05

\title{
НЕКОТОРЫЕ ПОДХОДЫ К РЕШЕНИЮ ЗАДАЧ ЛИНЕЙНОЙ МНОГОКРИТЕРИАЛЬНОЙ ОПТИМИЗАЦИИ
}

\author{
(c) 2021 г. $\quad$ Е. М. АРИСТОВА
}

\begin{abstract}
АннотАция. Статья содержит описание трех подходов к решению задачи линейной многокритериальной оптимизации: метод свертывания векторного критерия в суперкритерий, принцип приближения по всем локальным критериям к идеальному решению и метод, построенный на основе введения меры конфликта между целевыми критериями.
\end{abstract}

Ключевые слова: оптимальное по Парето решение, многокритериальная оптимизация, градиент, целевая функция, коэффициент взаимодействия, матрица взаимодействия, кооперация, конфликт, принцип большинства, правило принятия решений.

\section{SOME APPROACHES TO LINEAR MULTICRITERIA OPTIMIZATION PROBLEMS}

\author{
(c) $2021 \quad$ E. M. ARISTOVA
}

\begin{abstract}
In this paper, we describe three approaches to problems of linear multicriteria optimization: the method of folding a vector criterion into a super criterion, the approximation principle an ideal solution, and the method based on the conflict measure between target criteria.

Keywords and phrases: Pareto optimal solution, multicriteria optimization, gradient, objective function, coefficient of interaction, matrix of interaction, cooperation, conflict, majority principle, rule of making decisions.
\end{abstract}

AMS Subject Classification: 62C86, 90C05

1. Введение. Методы решения задач математического программирования с одним критерием интенсивно разрабатывались последние пятьдесят лет. Изучение таких методов, однако, отражало самый ранний и простой этап в развитии математического программирования. Жизнь оказалась сложнее. По мере того, как мы постепенно вступаем в век информатики, становится ясно, что практически любая серьезная реальная задача характеризуется больше чем одним критерием. Лица, принимающие решения, в значительно большей степени, чем когда бы то ни было, ощущают необходимость оценивать альтернативные решения с точки зрения нескольких критериев.

Результаты исследований задач планирования и управления показывают, что в реальной постановке эти задачи являются многокритериальными. Раннее при исследовании проблемы многокритериальности часто все критерии, кроме одного, выбранного доминирующим, принимались в качестве ограничений, оптимизация проводилась по доминирующему критерию. Такой подход к решению практических задач значительно снижает эффективность принимаемых решений. 
Многокритериальность является особенностью реальных систем, при этом критерии, которые необходимо оптимизировать, зачастую противоречат друг другу. Универсальным способом борьбы с многокритериальностью является переход к обобщенному критерию, который и оптимизируется. В основе такого перехода лежит использование различных функций агрегирования, причем результат не всегда поддается интерпретации, т. к. агрегирование вводится искусственным образом, поэтому возникает вопрос об адекватности полученного оптимального решения $[6,7]$.

Как правило, в задачах многокритериальной оптимизации предполагается, что все критерии независимы. Однако в большинстве реальных задач целевые функции почти неизбежно являются противоречивыми, конфликтующими. Отказ от учета этого фактора приводит к значительному упрощению задач, так что решения, полученные традиционными методами, представляют лишь незначительный интерес.

В данной статье предлагается несколько подходов к решению задач линейной многокритериальной оптимизации: метод свертывания критериев в суперкритерий (при одинаковой и разной важности критериев), принцип приближения по всем локальным критериям к идеальному решению и метод на основе введения меры конфликта между критериями и использования ее для задания стратегии агрегирования.

2. Постановка задачи. Дана задача многокритериальной оптимизации следующего вида:

$$
f_{1}(x) \rightarrow \max , \quad \ldots, \quad f_{n}(x) \rightarrow \max , \quad x \in X,
$$

где $x=\left(x_{1}, \ldots, x_{n}\right)^{T} \in \mathbb{R}^{n}, X \subseteq \mathbb{R}^{n}$ множество допустимых решений задачи $(1), f_{i}: \mathbb{R}^{n} \rightarrow \mathbb{R}, \forall i=$ $\overline{1, n}$ целевые функции (критерии), тах означает, что данный критерий нужно максимизировать.

Необходимо решить задачу (1) тремя указанными выше методами.

По существу многокритериальная задача отличается от обычной задачи оптимизации только наличием нескольких целевых функций вместо одной.

В задачах выбора решения, формализуемых в виде модели векторной оптимизации, первым естественным шагом следует считать выделение области компромиссов решений, оптимальных по Парето.

Вектор $x \in X$ называется оптимальным по Парето решением в задаче (1), если не существует $x^{0} \in X$ такого, что выполнены неравенства $f\left(x^{0}\right) \geqslant f(x)$ и $f\left(x^{0}\right) \neq f(x)$.

Областью компромиссов называется подмножество допустимого множества решений $X$, обладающего тем свойством, что все принадлежащие ему решения не могут быть улучшены одновременно по всем локальным критериям [3].

Оптимальное решение, выбираемое на основе многокритериального подхода, независимо от избираемого принципа оптимальности, всегда должно принадлежать области компромиссов. Иначе оно может быть улучшено и, следовательно, не является оптимальным.

Таким образом, область компромиссов есть область потенциально оптимальных компромиссов. Отсюда следует, что при выборе решения по векторному критерию эффективности можно ограничить поиск оптимального решения областью компромиссов, которая, как правило, значительно уже всей области возможных решений $X$.

3. Подходы к решению задачи многокритериальной оптимизации. Остановимся более подробно на трех методах решения задачи многокритериальной оптимизации: методе свертывания критериев в суперкритерий (при одинаковой и разной важности критериев), принципе приближения по всем локальным критериям к идеальному решению и методе, основанном на введении меры конфликта между критериями и использовании ее для задания стратегии агрегирования.

4. Подход, основанный на свертывании критериев в суперкритерий. Одним из распространенных методов решения многокритериальных задач является метод сведения многокритериальной задачи к однокритериальной путем свертывания векторного критерия в суперкритерий [1]. При этом каждый критерий умножается на соответствующий ему весовой коэффициент (коэффициент важности): 


$$
\Phi(x)=\sum_{i=1}^{n} \beta_{i} f_{i}(x) \text { при } \beta_{i} \geqslant 0, i=\overline{1, n} .
$$

При этом возникают трудности с правильным подбором весовых коэффициентов $\alpha_{i}$. Существуют различные способы выбора этих коэффициентов. Одним из них является назначение $\alpha_{i}$ в зависимости от относительной важности критериев (см. [9]):

\begin{tabular}{|l|c|}
\hline \multicolumn{1}{|c|}{ Определение } & $\begin{array}{c}\text { Интенсивность } \\
\text { относительной } \\
\text { важности }\end{array}$ \\
\hline Равная важность сравниваемых требований & 1 \\
\hline Умеренное(слабое) превосходство одного над другим & 3 \\
\hline Сильное(существенное) превосходство & 5 \\
\hline Очевидное превосходство & 7 \\
\hline Абсолютное(подавляющее) превосходство & 9 \\
\hline Промежуточные решения между двумя соседними оценками & $2,4,6,8$ \\
\hline
\end{tabular}

5. Принцип приближения по всем локальным критериям к идеальному решению. В основу данного метода положена идея приближения по всем критериям [2]. Пусть дана задача многокритериальной оптимизации вида (1). Среди решений этой системы требуется отыскать такое значение вектора $x^{*}=\left(x_{1}^{*}, \ldots, x_{k}^{*}\right)$, которое максимизирует как можно больше критериев [4].

Рассмотрим каждую отдельную функцию $f_{i}(x), i=\overline{1, n}$ и решим для нее задачу максимизации. Пусть соответствующие оптимальные планы это векторы

$$
x_{i}^{*}=\left(x_{1}^{*}, \ldots, x_{k}^{*}\right), \quad i=\overline{1, n} .
$$

На этих оптимальных планах определим значения критериев, получив вектор целевых функций на совокупности оптимальных решений

$$
f^{*}=\left(f_{1}\left(x_{1}^{*}\right), f_{2}\left(x_{2}^{*}\right), \ldots, f_{n}\left(x_{n}^{*}\right)\right) .
$$

Заметим, что для некоторых задач оптимальные планы могут совпадать, но отличаться значениями целевых функций. Составим вектор $F^{0}$ «идеальных» значений целевых функций $F^{0}=\left(F_{1}^{0}, \ldots, F_{n}^{0}\right)$ такой, что $F_{i}^{0}=f_{i}\left(x_{i}^{*}\right), i=\overline{1, n}$.

Будем искать такое решение задачи (1), которое минимизирует расстояние между вектором $f(x)$, содержащим текущие значения целевых функций, и вектором $F^{0}$. Обозначим квадрат евклидовой нормы вектора $f(x)-F^{0}$, определенного для всех $x \in X$ через

$$
R(x)=\left\|f(x)-F^{0}\right\|^{2} .
$$

Теперь поставленную задачу можно сформулировать в виде: дана задача многокритериальной оптимизации (1). Требуется определить точку $x \in X$, в которой функция $R(x)$ достигает своего минимума.

Таким образом, отыскание оптимального плана $x^{*} \in X$ сведено к оптимизации выражения (5) на множестве системы линейных неравенств задачи (1). Поскольку выражение (5) представляет собой квадратичную функцию переменных $x_{1}, \ldots, x_{n}$, то задача отыскания $x^{*}$ свелась к задаче выпуклого программирования: задана выпуклая функция $R(x)$, определенная на множестве $x \in$ $X$. Требуется отыскать точку $x^{*} \in X$, минимизирующую (5), т.е. обеспечивающую выполнение условия

$$
R\left(x^{*}\right)=\min _{x} R(x), \quad x \in X .
$$

Таким образом, алгоритм решения задачи (1) состоит из двух основных этапов: на первом осуществляется максимизация $F_{i}(x), i=\overline{1, k}$, а на втором минимизация $R(x)$. 


\section{6. Подход, основанный на введении меры конфликта между критериями.}

6.1. Анализ взаимодействия целевых функиий. Рассмотрим задачу многокритериальной оптимизации вида (1). Здесь $x=\left(x_{1}, \ldots, x_{n}\right)^{T} \in \mathbb{R}^{n}, X \subseteq \mathbb{R}^{n}$ множество допустимых решений задачи (1), $f_{i}: \mathbb{R}^{n} \rightarrow \mathbb{R}, \forall i=\overline{1, n}$ целевые функции (критерии).

Будем считать, что все функции $f_{i}(i=\overline{1, n})$ являются непрерывно дифференцируемыми в $X$, тогда для каждой целевой функции определен градиент в любой точке $x \in X$ :

$$
\nabla f_{i}(x)=\left(\frac{\partial f_{i}(x)}{\partial x_{1}}, \ldots, \frac{\partial f_{i}(x)}{\partial x_{n}}\right)^{T}
$$

Этот градиент представляет собой вектор, указывающий направление, в котором значение целевой функции увеличивается.

Заметим, что целевые функции в задаче (1) могут взаимодействовать различным образом. K основным типам взаимодействия можно отнести кооперацию, конфликт и независимость.

Пусть $f_{i}(x)$ и $f_{j}(x)$ две произвольные целевые функции из (1). Будем говорить, что

$(1)$ цель $f_{i}(x)$ кооперирует с целью $f_{j}(x)$, если

$$
\forall x^{\prime} \forall x^{\prime \prime}\left(f_{i}\left(x^{\prime \prime}\right) \geqslant f_{i}\left(x^{\prime}\right)\right) \Rightarrow\left(f_{j}\left(x^{\prime \prime}\right) \geqslant f_{j}\left(x^{\prime}\right)\right) ;
$$

(2) цель $f_{i}(x)$ конфликтует с целью $f_{j}(x)$, если

$$
\forall x^{\prime} \forall x^{\prime \prime}\left(f_{i}\left(x^{\prime \prime}\right) \geqslant f_{i}\left(x^{\prime}\right)\right) \Rightarrow\left(f_{j}\left(x^{\prime \prime}\right) \leqslant f_{j}\left(x^{\prime}\right)\right) ;
$$

(3) если предыдущие условия не выполняются, то целевые функции $f_{i}(x)$ и $f_{j}(x)$ независимы в $X$.

В случае кооперации достижение одной цели способствует достижению другой. Очевидно, что в этом случае эффект от достижения обеих целей превышает эффекты от каждой цели, взятой в отдельности. При конфликте целей достижение одной из целей приводит к тому, что другая цель не может быть достигнута. Также возможно, что тип взаимодействия динамически меняется в зависимости от значений целевых функций.

Так как все функции $f_{i}(i=\overline{1, n})$ непрерывно дифференцируемы в $X$, то для любой точки $x^{0}$ из $X$ и произвольного ненулевого приращения $p \in \mathbb{R}^{n}$ можно определить производную по направлению $p$ от функции $f_{i}(x)$ в точке $x^{0}$. Если направление $p$ задать с помощью единичного вектора с координатами $e=\left(\cos \alpha_{1}, \ldots, \cos \alpha_{n}\right)$, то производная в точке $x^{0}$ по направлению, определяемому вектором $e$, определяется как скалярное произведение векторов $\nabla f_{i}\left(x^{0}\right)$ и $e$, т.е.

$$
\frac{\partial f_{i}\left(x^{0}\right)}{\partial e}=\left(\nabla f_{i}\left(x^{0}\right), e\right)
$$

Заметим, что

(1) производная функции $f_{i}(x)$ в точке $x^{0}$ по направлению, определяемому градиентом этой функции в указанной точке, имеет максимальное значение по сравнению с производной в этой точке по любому другому направлению;

(2) значение производной функции $f_{i}(x)$ по направлению, определяемому градиентом этой функции в точке $x^{0}$, равно $\left|\nabla f_{i}\left(x^{0}\right)\right|$, т.е. длине вектора $\nabla f_{i}\left(x^{0}\right)$ в данной точке.

В самом деле,

$$
\frac{\partial f_{i}\left(x^{0}\right)}{\partial e}=\left(\nabla f_{i}\left(x^{0}\right), e\right)=\left|\nabla f_{i}\left(x^{0}\right)\right| \cdot|e| \cdot \cos \varphi,
$$

где $\varphi \in[0, \pi]$ угол между векторами $\nabla f_{i}\left(x^{0}\right)$ и $e$. Так как $|e|=1$, то

$$
\frac{\partial f_{i}\left(x^{0}\right)}{\partial e}=\left|\nabla f_{i}\left(x^{0}\right)\right| \cdot \cos \varphi
$$

Максимальное значение производной $\partial f_{i}\left(x^{0}\right) / \partial e$ имеем при $\cos \varphi=1$, т.е. при совпадении направления $е$ с направлением $\nabla f_{i}\left(x^{0}\right)$, причем производная по этому направлению будет равна $\left|\nabla f_{i}\left(x^{0}\right)\right|$. 
Рассмотрим две целевые функции $f_{i}(x)$ и $f_{j}(x)$, градиенты которых соответственно задаются векторами

$$
\nabla f_{i}(x)=\left(\frac{\partial f_{i}(x)}{\partial x_{1}}, \ldots, \frac{\partial f_{i}(x)}{\partial x_{n}}\right)^{T}, \quad \nabla f_{j}(x)=\left(\frac{\partial f_{j}(x)}{\partial x_{1}}, \ldots, \frac{\partial f_{j}(x)}{\partial x_{n}}\right)^{T} .
$$

Определим направление $e_{j}$, задаваемое вектором $\nabla f_{j}(x)$, в некоторой точке $x^{0}$ в виде

$$
e_{j}=\left(\frac{\partial f_{j}\left(x^{0}\right)}{\partial x_{1}} / \sqrt{\sum_{k=1}^{n}\left(\frac{\partial f_{j}\left(x^{0}\right)}{\partial x_{k}}\right)^{2}}, \ldots, \frac{\partial f_{j}\left(x^{0}\right)}{\partial x_{n}} / \sqrt{\sum_{k=1}^{n}\left(\frac{\partial f_{j}\left(x^{0}\right)}{\partial x_{k}}\right)^{2}}\right)^{T}=\widetilde{\nabla} f_{j}\left(x^{0}\right) .
$$

Заметим, что $\left|e_{j}\right|=\left|\widetilde{\nabla} f_{j}\left(x^{0}\right)\right|=1$, так что координаты представляют собой направляющие косинусы градиента функции $f_{j}(x)$ в точке $x^{0}$.

Найдем производную по направлению $e_{j}$ от целевой функции $f_{i}(x)$ в некоторой точке $x^{0}$.

$$
\begin{aligned}
\frac{\partial f_{i}\left(x^{0}\right)}{\partial e_{j}}=\left(\nabla f_{i}\left(x^{0}\right), e_{j}\right)=\sum_{r=1}^{n} & \frac{\partial f_{i}\left(x^{0}\right)}{\partial x_{r}} \cdot \frac{\frac{\partial f_{j}\left(x^{0}\right)}{\partial x_{r}}}{\sqrt{\sum_{k=1}^{n}\left(\frac{\partial f_{j}\left(x^{0}\right)}{\partial x_{k}}\right)^{2}}}= \\
& =\frac{1}{\sqrt{\sum_{k=1}^{n}\left(\frac{\partial f_{j}\left(x^{0}\right)}{\partial x_{k}}\right)^{2}}} \cdot \sum_{r=1}^{n} \frac{\partial f_{i}\left(x^{0}\right)}{\partial x_{r}} \cdot \frac{\partial f_{j}\left(x^{0}\right)}{\partial x_{r}}=\frac{\left(\nabla f_{i}\left(x^{0}\right), \nabla f_{j}\left(x^{0}\right)\right)}{\left|\nabla f_{j}\left(x^{0}\right)\right|} .
\end{aligned}
$$

Аналогично можно определить производную целевой функции $f_{j}(x)$ по направлению $e_{i}=\widetilde{\nabla} f_{i}\left(x^{0}\right)$, задаваемому градиентом $\nabla f_{j}(x)$ в некоторой точке $x^{0}$.

$$
\frac{\partial f_{j}\left(x^{0}\right)}{\partial e_{i}}=\left(\nabla f_{j}\left(x^{0}\right), e_{i}\right)=\frac{1}{\sqrt{\sum_{k=1}^{n}\left(\frac{\partial f_{i}\left(x^{0}\right)}{\partial x_{k}}\right)^{2}}} \cdot \sum_{r=1}^{n} \frac{\partial f_{i}\left(x^{0}\right)}{\partial x_{r}} \cdot \frac{\partial f_{j}\left(x^{0}\right)}{\partial x_{r}}=\frac{\left(\nabla f_{i}\left(x^{0}\right), \nabla f_{j}\left(x^{0}\right)\right)}{\left|\nabla f_{i}\left(x^{0}\right)\right|} .
$$

Заметим, что

$$
\frac{\partial f_{i}\left(x^{0}\right)}{\partial e_{j}} / \frac{\partial f_{j}\left(x^{0}\right)}{\partial e_{i}}=\frac{\left|\nabla f_{i}\left(x^{0}\right)\right|}{\left|\nabla f_{j}\left(x^{0}\right)\right|}
$$

Таким образом, для функции $f_{i}(x)$ имеем производную $\frac{\partial f_{i}\left(x^{0}\right)}{\partial e_{j}}$ по направлению $e_{j}$ в точке $x^{0}$, при этом ее максимальное значение $\left|\nabla f_{i}\left(x^{0}\right)\right|$ достигается в направлении, задаваемом градиентом $\nabla f_{i}\left(x^{0}\right)$ в этой же точке. Следовательно, для оценки отклонения направления $e_{j}$ от направления, соответствующего градиенту $\nabla f_{i}\left(x^{0}\right)$, можно использовать величину

$$
\begin{aligned}
\left.k_{j}\left(f_{i}\right)\right|_{x^{0}}=\frac{1}{\left|\nabla f_{i}\left(x^{0}\right)\right|} \frac{\partial f_{i}\left(x^{0}\right)}{\partial e_{j}}= & \frac{\sum_{r=1}^{n} \frac{\partial f_{i}\left(x^{0}\right)}{\partial x_{r}} \cdot \frac{\partial f_{j}\left(x^{0}\right)}{\partial x_{r}}}{\sqrt{\sum_{k=1}^{n}\left(\frac{\partial f_{i}\left(x^{0}\right)}{\partial x_{k}}\right)^{2}} \cdot \sqrt{\sum_{k=1}^{n}\left(\frac{\partial f_{j}\left(x^{0}\right)}{\partial x_{k}}\right)^{2}}}= \\
& =\sum_{r=1}^{n} \frac{\frac{\partial f_{i}\left(x^{0}\right)}{\partial e_{j}}}{\sqrt{\sum_{k=1}^{n}\left(\frac{\partial f_{i}\left(x^{0}\right)}{\partial x_{k}}\right)^{2}}} \cdot \frac{\frac{\partial f_{j}\left(x^{0}\right)}{\partial e_{j}}}{\sqrt{\sum_{k=1}^{n}\left(\frac{\partial f_{j}\left(x^{0}\right)}{\partial x_{k}}\right)^{2}}} .
\end{aligned}
$$


С другой стороны,

$$
\left.k_{j}\left(f_{i}\right)\right|_{x^{0}}=\frac{1}{\left|\nabla f_{i}\left(x^{0}\right)\right|} \frac{\partial f_{i}\left(x^{0}\right)}{\partial e_{j}}=\frac{\left(\nabla f_{i}\left(x^{0}\right), \nabla f_{j}\left(x^{0}\right)\right)}{\left|\nabla f_{i}\left(x^{0}\right)\right| \cdot\left|\nabla f_{j}\left(x^{0}\right)\right|}=\cos \varphi,
$$

где $\varphi \in[0, \pi]$ угол, образованный градиентами $\nabla f_{i}\left(x^{0}\right)$ и $\nabla f_{j}\left(x^{0}\right)$ в некоторой точке $x^{0}$.

Если $\cos \varphi>0$, то $\varphi$ острый угол (и можно говорить о кооперации соответствующих целевых функций $)$, если $\cos \varphi=0$, то $\varphi=\pi / 2$, т.е. градиенты образуют прямой угол, тогда цели будем считать независимыми, если же $\cos \varphi<0$, то угол $\varphi$ является тупым (и можно говорить о конфликте соответствующих целевых функций), поскольку $\varphi=\pi$ при $\cos \varphi=-1$ и векторы $\nabla f_{i}\left(x^{0}\right)$ и $\nabla f_{j}\left(x^{0}\right)$ противоположно направлены. Если $\cos \varphi=1$, то $\varphi=0$, и векторы $\nabla f_{i}\left(x^{0}\right)$ и $\nabla f_{j}\left(x^{0}\right)$ одинаково направлены.

Рассмотрим некоторые свойства введенного коэффициента, предполагая, что он вычислен в некоторой точке $x^{0}$.

$\left.k_{j}\left(f_{i}\right)\right|_{x^{0}}=\left.k_{i}\left(f_{j}\right)\right|_{x^{0}}$, поэтому в дальнейшем коэффициент взаимодействия целевых функций $f_{i}(x)$ и $f_{j}(x)$ в некоторой точке $x^{0}$ будем обозначать $k_{i j}\left(x^{0}\right)=k_{j i}\left(x^{0}\right)$. Заметим, что

$$
k_{i i}\left(x^{0}\right)=\frac{1}{\left|\nabla f_{i}\left(x^{0}\right)\right|} \frac{\partial f_{i}\left(x^{0}\right)}{\partial e_{j}}=\frac{\left(\nabla f_{i}\left(x^{0}\right), \nabla f_{i}\left(x^{0}\right)\right)}{\left|\nabla f_{i}\left(x^{0}\right)\right| \cdot\left|\nabla f_{i}\left(x^{0}\right)\right|}=\cos 0=1 .
$$

6.2. Задачи линейной оптимизачии. Рассмотрим частный случай задачи (1), когда все целевые функции линейны, т.е.

$$
f_{i}(x)=\sum_{p=1}^{k} c_{i p} x_{p}, \quad i=\overline{1, n},
$$

где $x=\left(x_{1}, \ldots, x_{n}\right)^{T} \in X \subseteq \mathbb{R}^{n}, c_{i}=\left(c_{i 1}, \ldots, c_{i k}\right)^{T}, i=\overline{1, n}, \quad$ вектор коэффициентов целевой функции.

В линейном случае градиент каждой целевой функции полностью определяется коэффициентом целевой функции $\nabla f_{i}(x)=c_{i}$ и представляет собой константу.

Коэффиииент взаимодействия иелевых функиий [1] определяется по формуле

$$
k_{i j}=\frac{\left(c_{i}, c_{j}\right)}{\left|c_{i}\right|\left|c_{j}\right|}=\left(\sum_{k=1}^{n} c_{i k} c_{j k}\right) /\left(\sqrt{\sum_{l=1}^{n} c_{i l}^{2}} \cdot \sqrt{\sum_{l=1}^{n} c_{j l}^{2}}\right) .
$$

Для определения типа взаимодействия разобьем промежуток $[0, \pi]$ на три промежутка

$$
[0, \pi]=\left[0, \frac{\pi}{3}\right] \cup\left(\frac{\pi}{3}, \frac{2 \pi}{3}\right) \cup\left[\frac{2 \pi}{3}, \pi\right] .
$$

Разбиение можно осуществить иначе, главное, чтобы получаемые промежутки поддавались интерпретации, и на их основе можно было бы строить качественные оценки взаимодействия целей.

С учетом введенного разбиения на основе коэффициентов $k_{i j}$ можно сформулировать следующие правила принятия решений:

(1) чем ближе $k_{i j}$ к 1 , тем в большей степени целевые функции $f_{i}(x)$ и $f_{j}(x)$ являются кооперирующими, поэтому если $k_{i j} \in[1 / 2,1]$, то цели кооперируют;

(2) чем ближе $k_{i j}$ к -1 , тем в большей степени цели $f_{i}(x)$ и $f_{j}(x)$ являются конкурирующими, поэтому если $k_{i j} \in[-1,-1 / 2]$, то цели конкурируют;

(3) чем ближе $k_{i j}$ к 0 , тем в большей степени цели $f_{i}(x)$ и $f_{j}(x)$ являются независимыми, поэтому если $k_{i j} \in[-1 / 2,1 / 2]$, то цели независимы.

Вычислив коэффициенты взаимодействия для каждой пары целевых функций, можно сформировать матрицу $K=\left\{k_{i j}\right\}_{N \times N}$ с элементами $\left|k_{i j}\right| \leqslant 1$, которая задает симметричное бинарное отношение. На его основе можно сформировать различные подходы.

Знак коэффициента позволяет сделать вывод о типе взаимодействия, при этом важна и количественная оценка такого взаимодействия. Для оценки силы взаимодействия целей воспользуемся понятием нечеткого множества. В этом случае каждому из промежутков $[0, \pi / 3],(\pi / 3,2 \pi / 3)$, 
$[2 \pi / 3, \pi]$ ставится в соответствие промежуток изменения $\cos \varphi$. Соответственно, имеем $[1 / 2,1]$, $(-1 / 2,1 / 2),[-1,-1 / 2]$.

6.3. Алгоритм решения задачи линейной многокритериальной оптимизации. Пусть задана задача линейной многокритериальной оптимизации в виде:

$$
f_{p}(x)=\sum_{i=1}^{2} c_{i}^{p} x_{i}^{p} \rightarrow \max \quad(p=\overline{1, n}),
$$

при $x \in X \subseteq \mathbb{R}^{n}$, где $X$ множество допустимых значений переменной $x, n$ число целевых функций (критериев). Предлагается следующий алгоритм решения этой задачи, построенный на основе введения меры конфликта между целевыми критериями и учитывающий тип их взаимодействия [1,5]:

(1) Для каждой целевой функции решить задачу максимизации с исходной допустимой областью, получив оптимальное решение $x_{p}^{*}$ и соответствующее значение целевой функции $f_{p}\left(x_{p}^{*}\right)$.

(2) Для каждой пары $f_{i}$ и $f_{j}$ целевых функций определить коэффициент взаимодействия $k_{i j}$ по формуле (13). Составить матрицу $K=\left\{k_{i j}\right\}_{n \times n}$ коэффициентов взаимодействия целевых функций.

(3) Определить тип взаимодействия между всеми парами целевых функций, используя для принятия решения правила:

(i) если $k_{i j} \in[1 / 2,1]$, то цели $f_{i}$ и $f_{j}$ кооперируют;

(ii) если $k_{i j} \in[-1,-1 / 2]$, то цели $f_{i}$ и $f_{j}$ конкурируют;

(iii) если $k_{i j} \in[-1 / 2,1 / 2]$, то цели $f_{i}$ и $f_{j}$ независимы.

Составить таблицу типов взаимодействия:

\begin{tabular}{|c|c|c|c|}
\hline & Кооперация & Конфликт & Независимость \\
\hline$f_{j}(j=\overline{1, n})$ & $S_{1}^{j}$ & $S_{2}^{j}$ & $S_{3}^{j}$ \\
\hline
\end{tabular}

где $S_{1}^{j}, S_{2}^{j}, S_{3}^{j}$ количества функций, с которыми конкретная целевая функция $f_{j}$ кооперирует, конфликтует и независима соответственно, для каждого $j$.

(4) Определить коэффициенты значимости $\alpha_{i}^{j}(i=\overline{1,3}, j=\overline{1, n}) i$-го взаимодействия для целевой функции $f_{j}(x)$ в ее обобщенной оценке по формуле

$$
\alpha_{i}^{j}=S_{i}^{j} / n,
$$

где $S_{i}^{j} \in[0,1]$, и для любого $j$ выполняется условие

$$
\sum_{i=1}^{3} \alpha_{i}^{j}=1
$$

(5) Для выбранного принципа принятия группового решения (будем рассматривать принцип большинства) построить ранжирование $\left(x_{i_{1}}^{*}, \ldots, x_{i_{p}}^{*}\right)$ точек решений $x_{p}^{*}$ по предпочтительности в зависимости от значений целевых функций [8]. Соответствующим образом упорядочить целевые функции.

(6) Назначить коэффициенты зависимости для каждой пары целевых функций.

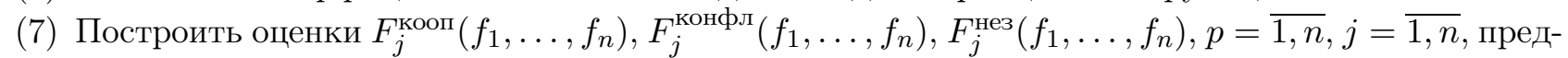
ставляющие собой сумму произведений функций, с которыми соответствующая $j$-я целевая функция кооперирует, конфликтует и независима, и коэффициентов ее зависимости с остальными целевыми функциями, входящими в конкретную оценку.

(8) Построить обобщенную целевую функцию по правилу:

$$
F\left(f_{1}, \ldots, f_{n}\right)=\sum_{j=1}^{n} F_{j}\left(f_{1}, \ldots, f_{n}\right),
$$

где

$$
F_{j}\left(f_{1}, \ldots, f_{n}\right)=\alpha_{1}^{j} F_{j}^{\text {кооп }}\left(f_{1}, \ldots, f_{n}\right)+\alpha_{2}^{j} F_{j}^{\text {конфл }}\left(f_{1}, \ldots, f_{n}\right)+\alpha_{3}^{j} F_{j}^{\text {нез }}\left(f_{1}, \ldots, f_{n}\right) .
$$


Учитывая определение весов, получим формулу:

$$
F\left(f_{1}, \ldots, f_{n}\right)=\frac{1}{n}\left(\sum_{i=1}^{n} s_{1}^{j} F_{j}^{\text {кооп }}\left(f_{1}, \ldots, f_{n}\right)+\sum_{i=1}^{n} s_{2}^{j} F_{j}^{\text {конфл }}\left(f_{1}, \ldots, f_{n}\right)+\sum_{i=1}^{n} s_{3}^{j} F_{j}^{\text {нез }}\left(f_{1}, \ldots, f_{n}\right)\right) .
$$

(9) Решить задачу математической оптимизации с обобщенной целевой функцией

$$
F\left(f_{1}, \ldots, f_{n}\right) \rightarrow \max , \quad x \in X \subseteq \mathbb{R}^{n} .
$$

7. Пример решения задачи. Рассмотрим следующую задачу линейной многокритериальной оптимизации:

при условиях

$$
\begin{aligned}
& f_{1}(x)=4 x_{1}+2 x_{2} \rightarrow \max \\
& f_{2}(x)=3 x_{1}+9 x_{2} \rightarrow \max \\
& f_{3}(x)=4 x_{1}-x_{2} \rightarrow \max \\
& f_{4}(x)=-2 x_{1}+4 x_{2} \rightarrow \max \\
& f_{5}(x)=-3 x_{1}+x_{2} \rightarrow \max
\end{aligned}
$$

$$
2 x_{1}+3 x_{2} \leqslant 18, \quad-x_{1}+3 x_{2} \leqslant 9, \quad 2 x_{1}-x_{2} \leqslant 10, \quad x_{1} \geqslant 0, \quad x_{2} \geqslant 0 .
$$

Решим задачу с помощью трех рассмотренных выше методов. Решим одноцелевые задачи при условиях(18), получив следующие результаты (3)-(4):

$$
\begin{aligned}
& x_{1}^{*}=(6,2) \Rightarrow f_{1}\left(x^{*}\right)=28, \\
& x_{2}^{*}=(3,4) \Rightarrow f_{2}\left(x^{*}\right)=45, \\
& x_{3}^{*}=(6,2) \Rightarrow f_{3}\left(x^{*}\right)=22, \\
& x_{4}^{*}=(0,3) \Rightarrow f_{4}\left(x^{*}\right)=12, \\
& x_{5}^{*}=(0,3) \Rightarrow f_{5}\left(x^{*}\right)=3 .
\end{aligned}
$$

Вначале применим метод свертывания критериев в суперкритерий. Рассмотрим две ситуации: (а) коэфбициенты важности критериев: $(1 / 5,1 / 5,1 / 5,1 / 5,1 / 5)$ (все критерии равнозначны). Тогда суперкритерий (2) имеет вид:

$$
\Phi(x)=\sum_{i=1}^{n} \beta_{i} f_{i}(x)=\sum_{i=1}^{n} \frac{1}{5} f_{i}(x)=\frac{1}{5} \sum_{i=1}^{n} f_{i}(x)=1,2 x_{1}+3 x_{2},
$$

при этом задача (17)-(18) сводится к максимизации $\Phi(x)$ при условиях (18). Решая эту задачу, получим ответ

$$
x^{*}=(3,4) ; \quad f_{1}^{*}=20, \quad f_{2}^{*}=45, \quad f_{3}^{*}=8, \quad f_{4}^{*}=10, \quad f_{5}^{*}=-5 .
$$

(b) коэффиииенты важсности критериев: $(3 / 10,3 / 10,2 / 10,1 / 10,1 / 10)$. Тогда суперкритерий (2) имеет вид:

$$
\Phi(x)=\sum_{i=1}^{n} \beta_{i} f_{i}(x)=2,4 x_{1}+3,6 x_{2},
$$

при этом задача (17)-(18) сводится к максимизации $\Phi(x)$ при условиях (18). Решая эту задачу, получим ответ

$$
x^{*}=(3,4) ; \quad f_{1}^{*}=20, \quad f_{2}^{*}=45, \quad f_{3}^{*}=8, \quad f_{4}^{*}=10, \quad f_{5}^{*}=-5 .
$$

Таким образом, используя метод свертывания критериев в суперкритерий, получаем, что наилучшим решением является точка $(3,4)$. Теперь рассмотрим приниип приближения по всем локальным критериям $к$ идеальному решению. Используя (19), заключаем, что вектор $F^{0}$ имеет вид $F^{0}=(28,45,22,13,3)$, и задача $(17)-(18)$ сводится к нахождению минимума функции $R(x)=\left\|f(x)-F^{0}\right\|^{2}$ при условиях (18): 


$$
\begin{aligned}
R(x)=\left(4 x_{1}+2 x_{2}-28\right)^{2}+\left(3 x_{1}+9 x_{2}-45\right)^{2}+\left(4 x_{1}-\right. & \left.x_{2}-22\right)^{2}+ \\
& +\left(-2 x_{1}+4 x_{2}-12\right)^{2}+\left(-3 x_{1}+x_{2}-3\right)^{2},
\end{aligned}
$$

решая которую, получаем ответ

$$
x^{*} \approx(3,66,3,56) ; \quad f_{1}^{*} \approx 21,75, \quad f_{2}^{*} \approx 43,03, \quad f_{3}^{*} \approx 11,06, \quad f_{4}^{*} \approx 6,94, \quad f_{5}^{*} \approx-7,41 .
$$

Таким образом, используя принцип приближения по всем локальным критериям к идеальному решению, получаем, что наилучшим решением является точка $(3,66 ; 3,56)$. И, наконец, рассмотрим метод, основанный на введении меры конфликта между иелевыми критериями. Определяя коэффициенты взаимодействия целевых функций по формуле (13), получим матрицу $K$ вида:

$$
K=\left(\begin{array}{rrrrr}
\sqrt{ } & 0,71 & 0,76 & 0,00 & -0,71 \\
0,71 & \sqrt{ } & 0,08 & 0,71 & -0,14 \\
0,76 & 0,08 & \sqrt{ } & -0,65 & -0,99 \\
0,00 & 0,71 & -0,65 & \sqrt{ } & 0,71 \\
-0,71 & -0,14 & -0,99 & 0,71 & \sqrt{ }
\end{array}\right)
$$

Составим таблицу типов взаимодействия целевых функций:

\begin{tabular}{|c|c|c|}
\hline Кооперация & Конфликт & Независимость \\
\hline$f_{1}(x)$ и $f_{2}(x)$ & $f_{1}(x)$ и $f_{5}(x)$ & $f_{1}(x)$ и $f_{4}(x)$ \\
\hline$f_{1}(x)$ и $f_{3}(x)$ & $f_{3}(x)$ и $f_{4}(x)$ & $f_{2}(x)$ и $f_{3}(x)$ \\
\hline$f_{2}(x)$ и $f_{4}(x)$ & $f_{3}(x)$ и $f_{5}(x)$ & $f_{2}(x)$ и $f_{5}(x)$ \\
\hline$f_{4}(x)$ и $f_{5}(x)$ & & \\
\hline
\end{tabular}

В случае кооперации достижение одной цели способствует достижению другой. При конфликте целей достижение одной из целей приводит к тому, что другая цель не может быть достигнута. В соответствии с алгоритмом, построим обобщенную целевую функцию. Вначале, используя матрицу взаимодействия целевых функций, построим следующую таблицу:

\begin{tabular}{|c|c|c|c|}
\hline \multirow{2}{*}{$\begin{array}{c}\text { Целевая } \\
\text { функция }\end{array}$} & \multicolumn{4}{|c|}{ Количество взаимодействующих функций } \\
\cline { 2 - 4 } & Кооперация & Конфликт & Независимость \\
\hline $\mathbf{1}$ & 3 & 1 & 1 \\
\hline $\mathbf{2}$ & 3 & 0 & 2 \\
\hline $\mathbf{3}$ & 2 & 2 & 1 \\
\hline $\mathbf{4}$ & 3 & 1 & 1 \\
\hline $\mathbf{5}$ & 2 & 2 & 1 \\
\hline
\end{tabular}

Таким образом, имеем матрицу коэффициентов значимости $\alpha_{i}^{j}(i=\overline{1,3}, j=\overline{1,5})$ :

$$
\alpha_{i}^{j}=\left(\begin{array}{ccccc}
3 / 5 & 3 / 5 & 2 / 5 & 3 / 5 & 2 / 5 \\
1 / 5 & 0 & 2 / 5 & 1 / 5 & 2 / 5 \\
1 / 5 & 2 / 5 & 1 / 5 & 1 / 5 & 1 / 5
\end{array}\right)^{T}
$$

Упорядочим точки $A, B, C$ решения однокритериальных задач для каждой целевой функции по убыванию их значений: 


\begin{tabular}{|l|r|r|r|r|}
\hline $\mathbf{f}_{\mathbf{1}}$ & 28 & 20 & 6 & $A B C$ \\
\hline $\mathbf{f}_{\mathbf{2}}$ & 36 & 45 & 27 & $B A C$ \\
\hline $\mathbf{f}_{\mathbf{3}}$ & 22 & 8 & -3 & $A B C$ \\
\hline $\mathbf{f}_{\mathbf{4}}$ & -4 & 10 & 12 & $C B A$ \\
\hline $\mathbf{f}_{\mathbf{5}}$ & -16 & -5 & 3 & $C B A$ \\
\hline & $\mathbf{A}(\mathbf{6}, \mathbf{2})$ & $\mathbf{B}(\mathbf{3}, \mathbf{4})$ & $\mathbf{C}(\mathbf{0}, \mathbf{3})$ & \\
\hline
\end{tabular}

В качестве принципа принятия группового решения будем использовать правило большинства: если дан профиль набор ранжировок, выражающих предпочтение членов группы $\left(p_{1}, \ldots, p_{t}\right)$, альтернатива $a$ получает в групповой ранжировке более высокое место, чем альтернатива $b$ тогда и только тогда, когда большинство (более половины) индивидуумов оценивают $a$ выше $b$ (см. [8]):

\begin{tabular}{|c|c|c|c|}
\hline & $\mathbf{1}$ & $\mathbf{2}$ & $\mathbf{3}$ \\
\hline $\mathbf{A}$ & 2 & 1 & 2 \\
\hline $\mathbf{B}$ & 1 & 4 & 0 \\
\hline $\mathbf{C}$ & 2 & 0 & 3 \\
\hline
\end{tabular}$\quad \Rightarrow$\begin{tabular}{|c|c|}
\hline $\mathbf{A}$ & 1,3 \\
\hline $\mathbf{B}$ & 2 \\
\hline $\mathbf{C}$ & 3 \\
\hline
\end{tabular}

Таким образом, ранжирование альтернатив представляет собой упорядоченную по убыванию важности последовательность точек $A B C$, которую запишем в виде:

$$
(6,2) \rightarrow(3,4) \rightarrow(0,3) .
$$

Следовательно, в соответствии с правилом большинства лучшей является точка $(6,2)$. Теперь применим правило большинства для построения обобщенной целевой функции. Используя коэффициенты значимости из (20) и ранжирование точек, полученное с помощью правила боль-

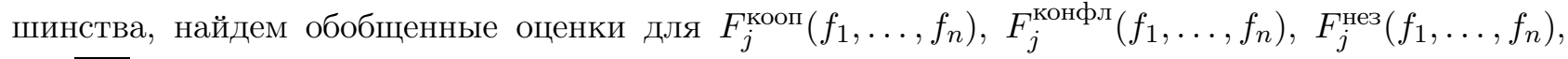
$j=\overline{1, n}$ :

$$
(1 \sim 3) \rightarrow(2) \rightarrow(4 \sim 5) .
$$

Правило принятия решений, например, имеет вид: функции, достигающие своего экстремума в одной точке, имеют коэффициент зависимости, равный 1. Функции, достигающие своего экстремума в соседних точках, в ранжировании точек имеют зависимость $0,5 \leftrightarrow 0,5$, в точках через две в ранжировании точек $0,7 \leftrightarrow 0,3$ и т. д., т.е. для нашей задачи

$$
(1 \sim 3) \stackrel{0,5 \leftrightarrow 0,5}{\longrightarrow}(2), \quad(1 \sim 3) \stackrel{0,7 \leftrightarrow 0,3}{\longrightarrow}(4 \sim 5), \quad(2) \stackrel{0,5 \leftrightarrow 0,5}{\longrightarrow}(4 \sim 5) .
$$

Для задачи определим функции, с которыми $j$-я целевая функция кооперирует, конфликтует и независима, записав их в следующую таблицу:

\begin{tabular}{|l|l|l|l|}
\hline $\mathbf{j}=\mathbf{1}$ & $G_{1}=\left\{f_{1}, f_{2}, f_{3}\right\}$ & $M_{1}=\left\{f_{5}\right\}$ & $N_{1}=\left\{f_{4}\right\}$ \\
\hline $\mathbf{j}=\mathbf{2}$ & $G_{2}=\left\{f_{1}, f_{4}\right\}$ & $M_{2}=\emptyset$ & $N_{2}=\left\{f_{3}, f_{5}\right\}$ \\
\hline $\mathbf{j}=\mathbf{3}$ & $G_{3}=\left\{f_{1}\right\}$ & $M_{3}=\left\{f_{4}, f_{5}\right\}$ & $N_{3}=\left\{f_{2}\right\}$ \\
\hline $\mathbf{j}=\mathbf{4}$ & $G_{4}=\left\{f_{2}, f_{5}\right\}$ & $M_{4}=\left\{f_{3}\right\}$ & $N_{4}=\left\{f_{1}\right\}$ \\
\hline $\mathbf{j}=\mathbf{5}$ & $G_{5}=\left\{f_{4}\right\}$ & $M_{5}=\left\{f_{1}, f_{3}\right\}$ & $N_{5}=\left\{f_{2}\right\}$ \\
\hline
\end{tabular}

Затем построим обобщенные оценки $F_{j}\left(f_{1}(x), \ldots, f_{n}(x)\right), j=\overline{1,5}$ по формуле (16) для целевых функций $f_{j}(x), j=\overline{1,5}$, в которых коэффициенты значимости имеют вид (20).

После этого определяется обобщенная целевая функция:

$$
F\left(f_{1}(x), \ldots, f_{5}(x)\right)=\sum_{j=1}^{5} F_{j}\left(f_{1}(x), \ldots, f_{5}(x)\right) .
$$

Проведя соответствующие преобразования, получим, что обобщенная функция примет вид:

$$
F\left(f_{1}(x), \ldots, f_{5}(x)\right)=3,94 x_{1}+3,28 x_{2} .
$$


Решая задачу линейного программирования с допустимой областью решений (18) и целевой функцией $(21)$, получим решение

$$
x^{*}=(6,2) ; \quad f_{1}^{*}=28, \quad f_{2}^{*}=36, \quad f_{3}^{*}=22, \quad f_{4}^{*}=-4, \quad f_{5}^{*}=-16 .
$$

Таким образом, используя принцип большинства для построения обобщенной целевой функции, получим, что наилучшим решением является точка $(6,2)$.

8. Заключение. Выбор оптимального решения, как правило, опирается на совокупность критериев, характеризующих эффективность, полезность, качество допустимых решений. В оптимизационных задачах формально критерий может быть представлен целевой функцией. Если при решении задач с одной целевой функцией методологических проблем не возникает, а возможны только вычислительные трудности, то иначе обстоит дело с задачами, в которых несколько целевых функций. Здесь основные нюансы связаны со следующей проблемой: что следует считать наилучшими решениями в задаче с несколькими целевыми функциями, которые, возможно, противоречивы и достигают максимума в различных точках множества решений? Процедура сведения многокритериальной задачи к однокритериальной в большинстве случаев приводит к серьезному искажению существа проблемы и, следовательно, к неоправданной замене одной задачи другой. В основе такого перехода лежит использование различных функций агрегирования, причем результат не всегда поддается интерпретации, т. к. агрегирование вводится искусственным образом, поэтому возникает вопрос об адекватности полученного оптимального решения. Стоит отметить, что цели в задачах с несколькими целевыми функциями могут находиться друг с другом в различных отношениях. В качестве основных типов взаимодействия целевых функций в задаче линейного программирования в работе выделены три кооперация, конфликт и независимость. В случае кооперации достижение одной цели способствует достижению другой. При конфликте целей достижение одной из целей приводит к тому, что другая цель не может быть достигнута. В статье рассматривается метод свертывания векторного критерия в суперкритерий в случае равной и разной важности коэффициентов относительной важности критериев, а также принцип приближения по всем локальным критериям к идеальному решению и метод, построенный на основе введения меры конфликта между целевыми критериями. Затем рассматривается задача, для решения которой предлагается использовать эти методы. Как видно из решения задачи, наиболее объективное решение дает метод, учитывающий взаимодействие между целевыми функциями в многокритериальной задаче.

\section{СПИСОК ЛИТЕРАТУРЫ}

1. Аристова E. M. Учет взаимодействия между целевыми функциями и их агрегирование в задачах оптимизации / Дисс. на соиск. уч. степ. канд. физ.-мат. наук. - Воронеж: Воронеж. гос. ун-т, 2012.

2. Борисов А. Н. Обработка нечеткой информации в системах принятия решений. - М.: Радио и связь, 1989.

3. Зайченко Ю. П. Исследование операций. Нечеткая оптимизация. - Киев: Выща школа, 1991.

4. Мелькумова E. M. Один из подходов к решению задачи многокритериальной оптимизации// Вестн. ВГУ. Сер. Сист. анал. информ. технол. - 2010. - 2. - С. 3942.

5. Мелькумова E. М. Решение задачи многокритериальной оптимизации с помощью учета коэффициентов важности целевых функций// в кн.: Актуальные проблемы прикладной математики, информатики и механики. - Воронеж: Изд-во ВГУ, 2011. - С. 265269.

6. Ногин В. Д. Принятие решений в многокритериальной среде. - М.: Физматлит, 2002.

7. Петровский А. Б. Теория принятия решений. - М.: Академия, 2009.

8. Робертс Ф. С. Дискретные математические модели с приложениями к социальным биологическим и экологическим задачам. - М.: Наука, 1986.

9. Саати Т. Принятие решений. Метод анализа иерархий. - М.: Радио и связь, 1993.

Аристова Екатерина Михайловна

Воронежский государственный университет

E-mail: pmim@yandex.ru 